\title{
Les petites écoles rurales en Finlande
}

Small rural schools in Finland

Las pequeñas escuelas rurales en Finlandia

\section{Arja-Sisko Holappa}

\section{OpenEdition}

\section{Journals}

Édition électronique

URL : http://journals.openedition.org/ries/2286

DOI : $10.4000 /$ ries.2286

ISSN : 2261-4265

Éditeur

Centre international d'études pédagogiques

Édition imprimée

Date de publication : 1 avril 2012

Pagination : 103-112

ISBN : 978-2-85420-594-7

ISSN : $1254-4590$

Référence électronique

Arja-Sisko Holappa, « Les petites écoles rurales en Finlande », Revue internationale d'éducation de Sèvres [En ligne], 59 | avril 2012, mis en ligne le 06 février 2015, consulté le 01 mai 2019. URL : http:// journals.openedition.org/ries/2286 ; DOI : 10.4000/ries.2286 


\section{Les petites écoles rurales en Finlande*}

\section{Arja-Sisko Holappa}

\section{QUELQUES DONNÉES CONCERNANT L'ENSEIGNEMENT OBLIGATOIRE EN FINLANDE}

La majorité de la population finlandaise, qui s'élève à 5,4 millions d'habitants, vit à proximité de la côte sud du pays. En termes de densité de population, l'est et le nord du pays sont les zones les plus rurales. Toutefois, du point de vue de l'enseignement obligatoire, il existe des petites écoles rurales dans l'ensemble du pays. En Finlande, le concept de ruralité en matière d'éducation fait référence à de petites écoles de vingt à quatre-vingts élèves et des classes à niveaux multiples, c'est-à-dire à une situation pédagogique dans laquelle les enseignants ont une possibilité limitée de partager leurs idées avec leurs collègues et de participer à une formation sur site. Du point de vue des enfants, la ruralité signifie de longues distances à parcourir pour parvenir jusqu'à l'école, quelques rares amis, des difficultés à participer à des activités extrascolaires, au tutorat ou à l'étude et à bénéficier de structures d'aide personnalisée. De nombreuses petites écoles rurales ont également été récemment fermées.

Selon les données de l'Institut finlandais de la statistique, le taux de natalité continue à s'élever légèrement. Cependant, il demeure sous le seuil permettant le renouvellement des générations. La population finlandaise s'accroît légèrement grâce à l'immigration. On s'attend à ce que cet accroissement perdure, même dans un avenir plus lointain. Les déplacements dans le pays et à l'intérieur des villes elles-mêmes se font généralement en direction des grandes villes ou des centres urbains. À cause de cette tendance de la population à se concentrer, il n'est pas toujours aisé d'assurer dans les zones rurales des services publics tels que la santé, les soins et l'éducation.

En 2010, le nombre d'enfants âgés de 7 à 15 ans s'élevait à 540000 et il y avait environ trois mille établissements scolaires obligatoires (écoles dispensant un enseignement fondamental). Environ $20 \%$ de ces établissements sont de petites écoles regroupant moins de cinquante élèves. L'enseignement fondamental est une obligation et l'ensemble du groupe d'âge de 7 à 16 ans doit être scolarisé, sous une forme ou sous une autre. Aux termes de la loi, l'enseignement

* Article traduit de l'anglais par Robert Elbaz. 
public obligatoire devrait être entièrement gratuit pour les élèves et leur famille. Ce principe concerne également les déplacements scolaires, les repas quotidiens et les services d'accompagnement et de soutien. Les parents sont libres du choix de l'école mais la plupart des enfants d'âge scolaire obligatoire vont à l'école publique la plus proche. Le nombre d'écoles privées est faible et très peu d'enfants sont scolarisés à domicile. Les enfants ayant des besoins spécifiques sont scolarisés dans des écoles générales, surtout s'ils habitent dans des zones rurales. Le nombre d'écoles spécialisées a récemment diminué en raison de la mise en place d'une politique d'intégration (PEPA 2011).

Dans les classes 1 à 6 , il y a généralement un enseignant unique, responsable de la plupart des cours. De la septième à la neuvième année, les enseignants gèrent chacun une matière particulière. Les élèves ayant des besoins spécifiques peuvent en bénéficier dans chaque école. Il existe également des enseignants spécialisés dans les zones rurales mais ils travaillent le plus souvent à temps partiel dans les petites écoles isolées. À l'heure actuelle, la qualification des enseignants est plutôt bonne sur l'ensemble du territoire mais on constate un manque de services de suivi psychologique et social des enfants et de leurs familles, tout spécialement dans les zones rurales. Il est également plus difficile d'obtenir un personnel d'assistance spécialisé dans les petites écoles. En dépit de ces problèmes, les habitants de ces zones rurales sont très fiers de leurs écoles et y sont particulièrement attachés. Parents et enseignants considèrent que les petits établissements constituent un environnement bienveillant et protecteur pour les jeunes enfants (Peltonen 2002 ; Kilpelainen 2010). Dans le débat politique local, on entend souvent dire que les petits établissements coûtent trop cher, sont démodés et stimulent insuffisamment les enfants. Les contre-arguments des chercheurs sont souvent passés sous silence (Korpinin 2010). Ce genre d'opinions peut être interprété comme étant le reflet de valeurs néolibérales, valeurs qui se sont renforcées au cours des dix dernières années, en Finlande comme partout ailleurs (Holappa 2007, 275-308).

\section{Évolution DE L'OPTIMISME ÉDUCATIF}

Tout au long de son histoire, la ruralité a toujours joué un rôle important dans le système éducatif finlandais. C'est la raison pour laquelle un bref retour en arrière s'impose. La première loi concernant l'éducation publique fondamentale finlandaise a été rédigée il y a presque cent cinquante ans. Pour un pays très pauvre, dépourvu d'industrie, c'était une tâche gigantesque que de mettre sur pied un système scolaire. Il n'y avait ni enseignants, ni bâtiments scolaires, ni routes desservant tous les villages, ni argent. En revanche, il y avait beaucoup de doutes sur l'intérêt d'offrir une éducation aux enfants des classes populaires. Ces doutes étaient partagés non seulement par l'élite politique mais par les parents eux-mêmes. En effet, les enfants constituaient une part non 
négligeable de la main-d'œuvre. La législation et l'idée largement répandue que l'éducation constituait un moyen de faire reculer la pauvreté et de cultiver les gens ont culturellement contribué à fonder des écoles dans l'ensemble du pays, mais il a fallu presque un siècle pour construire un système d'éducation fondamentale égalitaire.

Le nombre d'établissements assurant l'enseignement obligatoire en Finlande ne cesse de croître jusqu'aux années cinquante. La législation en matière scolaire ayant décrété qu'aucun enfant ne devait être éloigné d'une école de plus de cinq kilomètres, ce principe aboutit à la création d'écoles dans pratiquement tous les villages. Ces écoles offrent un enseignement primaire aux 4-6 ans dans les deux langues officielles, le finnois et le suédois. L'enseignement secondaire n'est accessible en pratique qu'aux seuls enfants des classes sociales supérieures.

Les années soixante sont une époque de changements sociétaux rapides. La société, autrefois agricole, se transforme rapidement pour donner naissance à un pays industriel moderne. Le taux de natalité chute et les migrations de population vident les campagnes. Le nombre d'écoles de village commence à décroître. Simultanément, l'optimisme éducatif va croissant. En 1970, une nouvelle loi sur l'enseignement fondamental définit une scolarité publique obligatoire de neuf ans pour tous. Cette scolarité obligatoire, plus longue, nécessite la construction de nouveaux bâtiments scolaires. Elle est divisée en deux niveaux : les établissements d'enseignement fondamental primaire, assurant les six premières années (de 7 à 11 ans) et les établissements d'enseignement fondamental secondaire, assurant les trois dernières années (de 12 à 15 ans). Ces divisions demeurent le modèle le plus répandu, même si de nombreuses comprehensive schools ${ }^{1}$, assurant donc les neuf années de scolarité, ont été fondées au cours des dix dernières années. La division de l'enseignement obligatoire en deux niveaux fait qu'il existe habituellement plusieurs écoles de type primaire dans chaque municipalité mais peu d'écoles de type secondaire, voire une seule.

L'optimisme éducatif est également à l'œuvre dans le développement de l'enseignement spécialisé. Il existe toujours des établissements recevant des enfants à besoins spécifiques, mais la politique de développement de l'intégration de ces élèves dans les classes normales continue de plus belle. Des supports d'apprentissage spécifiques sont également disponibles pour pallier les handicaps cognitifs mineurs. Grâce à ces systèmes de soutien, pratiquement tous les élèves mènent à bien leur scolarité obligatoire. Le système scolaire dans son ensemble est également assez efficace puisque son coût total se situe dans la moyenne internationale (OCDE 2011).

1. Écoles polyvalentes sur le modèle anglo-saxon, regroupant différentes filières et/ou différents stades éducatifs. $(N d T)$ 
Fin 2010, environ $60 \%$ des Finlandais avaient atteint un diplôme scolaire post-comprehensive (soit au-delà de la neuvième année) en suivant différents parcours : établissements éducatifs généraux secondaires, enseignement professionnel, enseignement polytechnique ou universitaire. Quarante ans plus tôt, ce nombre était bien inférieur. Fin 1970, seul un quart de la population âgée de 15 ans ou plus avait atteint ce même niveau. Les jeunes sont tout spécialement bien formés : environ $90 \%$ des jeunes du groupe d'âge allant jusqu'à 25 ans ont mené à terme des études correspondant au moins au niveau secondaire.

\section{DROIT FONDAMENTAL OU MESURE COÛTEUSE ?}

La Finlande continue d'accorder une grande place à l'éducation. Le dernier programme gouvernemental insiste sur son importance sociale, culturelle et financière. Mais cet optimisme diffère sensiblement de celui des premières décennies. Dans les années soixante-dix, les municipalités souhaitaient offrir le meilleur enseignement possible à leurs enfants. Même dans les campagnes, les bâtiments scolaires furent rénovés et de nouveaux équipements furent mis à la disposition de tous. Les plus petites municipalités offraient des cours de langues étrangères et des activités extrascolaires gratuites. L'État investissait dans la formation des enseignants. À partir du début des années quatre-vingt, tous les enseignants exerçant dans le cadre de l'enseignement obligatoire possédaient un master universitaire. De nos jours, l'éducation est fréquemment perçue comme un poste de dépenses pour la société et les contribuables. Ce changement d'attitude se manifeste également par le démantèlement des petits établissements. Entre 2004 et 2008, par exemple, le nombre d'élèves relevant de l'enseignement obligatoire a baissé de $5 \%$ et celui des écoles de $14 \%$. Les décisions de fermeture sont prises par les conseils municipaux et se voient essentiellement justifiées par des arguments économiques, même si les conclusions des études montrent que les petits établissements disposent de nombreux atouts pédagogiques (Peltonen 2002; Karlberg-Granlund 2009; Kilpeläinen 2010).

Le nombre total d'établissements assurant une scolarité obligatoire en Finlande a diminué de 40 \% depuis 1990, principalement dans les zones rurales. Cette évolution est liée aux changements démographiques dans ces mêmes zones. Plus de 80 \% des fermetures concernent les petites écoles de moins de cinquante élèves, situées dans de petits villages, au nord et à l'est du pays. À l'heure actuelle, le nombre d'établissements diminue tout particulièrement dans la partie centre-ouest du pays et la taille moyenne des écoles ainsi fermées n'a cessé d'augmenter. La moitié des élèves d'âge scolaire obligatoire va maintenant dans des écoles regroupant plus de trois cents élèves. 
Depuis 2007, les agences régionales d'administration d'État ont évalué l'accessibilité des élèves aux établissements assurant la scolarité obligatoire et les études secondaires dans différentes parties du pays. Les résultats montrent que $93 \%$ des élèves des écoles regroupant des classes de niveau 1 à 6 vivent à moins de cinq kilomètres de leur école. Parmi les élèves des classes 7 à 9 , ce pourcentage tombe à $75 \%$. Le taux total d'accessibilité demeure élevé car la plus grande partie de la population finlandaise est concentrée dans les villes. Il existe cependant des différences régionales. Dans la région de Kainuu, au nord-est de la Finlande, par exemple, seuls $81 \%$ des élèves les plus jeunes vivent à proximité de leur école. Si nous examinons maintenant les résultats de cette évaluation au niveau municipal, il existe des différences significatives. Il y a vingt municipalités en Finlande dans lesquelles il n'existe aucune école regroupant les niveaux 7 à 9. Dans ce cas, les élèves doivent se rendre à l'école dans une municipalité voisine (PEPA 2011).

Selon la loi sur l'enseignement fondamental, la municipalité doit assurer le transport des élèves d'âge scolaire qui habitent à plus de cinq kilomètres de leur établissement ou dont le trajet vers l'école est trop dangereux ou trop difficile. Cela signifie que $24 \%$ des élèves d'âge scolaire obligatoire utilisent un véhicule quotidiennement (Pepa 2010). Dans des zones rurales, presque tous les élèves viennent à l'école en car, en taxi ou en bateau. Pour l'instant, les déplacements scolaires les plus longs sont d'environ cent soixante kilomètres par jour, dans le nord de la Finlande. Cette loi limite également le temps maximum dévolu au transport scolaire. Le trajet aller-retour ne devrait pas prendre plus de deux heures trente par jour. Dans la plupart des zones rurales, il est difficile de respecter cette contrainte temporelle mais le nombre d'élèves concernés par des déplacements trop longs a récemment diminué, grâce à une meilleure planification des transports scolaires dans les municipalités. En dépit des longues distances, il n'existe que très peu de dortoirs ou d'internats en Finlande. La possibilité de vivre chez soi est considérée comme un droit pour les enfants.

Il est vrai que l'enseignement dans de petites unités revient plus cher par élève que dans des établissements plus grands. En Finlande, la scolarité obligatoire est organisée par les municipalités mais la plus grande partie des coûts est financée par le gouvernement national. Cette subvention nationale compense en partie les coûts dus aux différences démographiques entre les municipalités. L'organisation de l'enseignement revient cependant toujours plus cher aux municipalités des zones rurales. Le traitement des enseignants est le même dans tout le pays mais les coûts d'enseignement dans les écoles les plus petites sont plus élevés en raison de groupes de classes plus restreints. Dans le nord de la Finlande, par exemple, les coûts par élève varient de 3960 euros par an en ville à 4616 euros par an dans les municipalités faiblement peuplées. En particulier, les coûts relatifs au transport scolaire, aux services de soutien social et psychologique et aux locaux sont plus élevés dans les zones rurales. En moyenne, le coût total de la scolarité obligatoire dans les municipalités à faible population est de 12 \% supérieur à celui des grandes agglomérations (Psavi 2011). 
Ce coût supérieur n'est qu'une facette du problème. Durant la période 2000-2004, selon les statistiques économiques de l'éducation, plus de trois cents petits établissements scolaires ont été fermés et, concomitamment, l'ensemble des coûts imputables à l'enseignement obligatoire a augmenté de $24 \%$ (Korpinen 2010). Le traitement des enseignants a augmenté mais cela n'a pas empêché la hausse totale des coûts d'être de $10 \%$ supérieure à l'indice moyen des prix à la consommation. Même si les municipalités font valoir des arguments économiques pour fermer les établissements, nombreuses sont celles qui ne sont pas parvenues à réduire leurs dépenses. Très souvent, les décisionnaires de ces municipalités ne s'intéressent qu'à leur propre district et aux conséquences à court terme. Ce manque de coopération entre municipalités a généré une augmentation des coûts de transport. Ces fermetures d'écoles ont tout spécialement affecté l'atmosphère dans les zones rurales, où la source de revenus repose sur le petit entreprenariat privé. Sans écoles, ces microsociétés cessent d'attirer les familles qui ont des enfants d'âge scolaire et se condamnent à terme à disparaître.

\section{L'AVENIR DES PETITES ÉCOLES RURALES EN FinLANDE}

Il existe toujours de nombreuses petites écoles rurales en Finlande de même que, en raison de la structure même du peuplement, il y aura également toujours des écoles urbaines à l'avenir. Cependant, le discours politique est contradictoire. D'une part l'égalité est considérée comme l'une des valeurs les plus importantes en matière d'éducation mais, d'autre part, fort peu d'attention a été accordée à l'accès à l'éducation dans les zones rurales.

L'accessibilité et la disponibilité de l'offre éducative sont considérées comme l'un des éléments clefs de l'État-providence scandinave. Tout récemment, on a critiqué le principe d' " une école pour tous ", mais les bons rapports d'évaluation indiquent que notre système scolaire fonctionne bien. Selon les résultats aux tests PISA, le contexte social n'a pas, en Finlande, autant d'influence sur les performances des élèves que dans de nombreux autres pays, et les différences entre établissements sont minimes (Kupiainen, Hautamaki et Karjalainen 2009). Ces résultats peuvent être considérés comme une preuve d'égalité éducative. Les parents peuvent être assurés que leurs enfants peuvent aussi recevoir la meilleure éducation possible dans les écoles rurales.

Les études qui concernent ces écoles montrent que la petite taille n'implique nullement des performances plus faibles. Par exemple, des recherches récentes menées par Kilpenainen (2010) font apparaitre que les petites écoles de village ont plus d'atouts que de faiblesses en termes d'enseignement et d'apprentissage. Les bâtiments scolaires et les aires de jeu sont fonctionnels et conçus pour les enfants. Les écoles peuvent utiliser la nature environnante de multiples 
façons dans le cadre de l'enseignement. L'atmosphère dans les écoles de village est encourageante et la coopération des enseignants avec les parents est tout à la fois naturelle et aisée. Selon Kilpelainen, les plus grosses difficultés sont liées aux longues distances et au manque de certaines structures telles que les équipements nécessaires pour se doucher après les activités physiques. Malgré ces inconvénients, enfants et parents apprécient leur école et leurs enseignants (Karlberg-Granlund 2009).

\section{LA VIE DANS LES PETITES ÉCOLES RURALES}

Il n'empêche que la vie quotidienne n'est pas toujours facile dans les petites écoles rurales. Malgré les résultats positifs des recherches conduites, le personnel des petites écoles ne reçoit pas suffisamment d'aide et d'encouragements pour développer une culture d'établissement. Jusqu'à présent, il a été relativement facile de recruter des enseignants qualifiés, même dans les zones rurales. À l'avenir, la situation ne sera certainement pas la même. La formation initiale des enseignants ne prend pas suffisamment en compte la pédagogie utilisée dans les petits établissements, telle que les méthodes d'apprentissage pour les groupes multi-âges. De même, aucun accompagnement valable à la prise de fonction ni aucune formation sur site ne sont disponibles. Les zones rurales ne tentent pas les nouveaux diplômés. Seules les municipalités ayant une identité culturelle forte et la possibilité d'employer les conjoints sont parvenues à attirer des enseignants.

Les enseignants en poste dans de petits établissements ont également besoin d'une aide pédagogique telle que la possibilité de partager leurs idées avec les enseignants d'autres établissements ou de participer à une formation sur site. Les nouveaux réseaux sociaux offrent des voies nouvelles pour l'interaction professionnelle. Des expériences prometteuses ont été conduites afin de créer des salles des professeurs virtuelles dans les écoles rurales. Des séances de formation virtuelles sur Internet ont également connu un grand succès. Assister à une formation traditionnelle sur site revient relativement cher. D'autre part, il est difficile de trouver des remplaçants, surtout pour des personnels affectés dans des écoles rurales. Pour l'instant, plusieurs municipalités participent au programme de développement appelé «Osaava». L’objectif principal de ce programme est de créer de nouveaux moyens pour gérer les compétences professionnelles des personnels d'enseignement et favoriser la coopération entre municipalités.

Les compétences professionnelles des enseignants sont un facteur de qualité important en matière d'éducation. Outre leur savoir-faire, les directives générales et les ressources ont leur importance. Le curriculum national constitue le cadre de référence le plus important pour les municipalités et les écoles locales (Holappa 2007). Le processus de mise à jour du curriculum au niveau national 
et local a récemment commencé. La précédente version de ce curriculum, qui date de 2004, n'avait pas pris en compte les variations au sein des différents types d'établissements scolaires finlandais. On a également oublié de tenir compte des petits établissements dans les processus locaux. Par exemple, les changements d'emploi du temps sont plus difficiles à mettre en place dans les petits établissements. Les atouts et les faiblesses des petites écoles devraient être pris en compte dans le texte du curriculum-cadre et dans les curricula municipaux.

Dans de nombreuses municipalités, la situation économique est préoccupante. Cela concerne également les petits établissements, souvent considérés comme un poste de dépenses supplémentaires. L'évaluation conduite par les agences régionales d'administration d'État (Pepa 2011) a montré que le réseau actuel des établissements devrait être maintenu en l'état, de façon à ce que les plus jeunes des enfants scolarisés dans les classes 1 à 6 puissent aller à l'école relativement près de chez eux. Maintenir la situation actuelle exige une coopération entre municipalités ainsi qu'une aide du gouvernement national.

« Les racines du succès finlandais aux tests PISA doivent être recherchées dans l'histoire et le développement rapide de l'État-providence finlandais tout autant que dans la politique éducative audacieuse conduite au cours des quarante dernières années, politique qui a mis l'accent sur l'égalité en matière d'éducation " (Kupiainen, Hautamaki ja Karjalainen 2009). Une nation très pauvre est parvenue à mettre en place un système scolaire qui couvre l'ensemble du pays, au service de toutes les familles. Il ne devrait pas être trop difficile pour l'État actuel d'assurer la pérennité de ce système. En ce qui concerne les petites écoles rurales, toutefois, la politique éducative finlandaise doit être rénovée. Les idées et les exemples de bonnes pratiques éducatives venant d'autres pays seront une aide précieuse dans notre travail d'amélioration.

\section{BibliographiE}

HOLAPPA A.-S. (2007) : Perusopetuksen opetussuunnitelma 2000-luvulla. [Le curriculum de l'éducation de base dans les années 2000. Processus de réforme au niveau local dans deux villes]. Thèse de doctorat E 94. Université d'Oulu.

KARLBERG-GRANLUND G. (2009) : Att förstå det stora $i$ det lilla. Byskolan som pedagogik, kultur och struktur. [Écoles rurales, pédagogie, culture et structure]. Thèse de doctorat. Åbo Academi.

KILPELAINEN, R. (2010) : Kyläkoulut Suomessa, maaseudun pienet koulut opettajien kuvaamina oppimis- ja kasvuympäristöinä. [Écoles rurales en Finlande, réflexions d'enseignants sur l'école comme environnement d'apprentissage à la campagne]. Thèse de doctorat. Université d'Oulu. 
KORPINEN E., ed. (2010) : Elakoon kylakoulu. [Hourra pour les écoles rurales]. PS-kustannus, Jyväskylä.

KUPIAINEN S., HAUTAMAKI J. et KARJALAINEN T. (2009): Finnish Education System and PISA [Le système éducatif finlandais et PISA]. Ministère de l'éducation, Finlande 209:46. Consultable en ligne sur : http://www.pisa2006.helsinki.fi/files/The_ Finnish_education_system_and_PISA.pdf

LAITILA T. (1999) : Siirtoja koulutuksen ohjauskentalla. Suomen yleissivistavan koulutuksen ohjaus 1980- ja 1990-luvuilla. [Les changements de la politique éducative. Le système de contrôle de l'éducation obligatoire finlandaise dans les années 1980 et 1990]. Thèse de doctorat. C 146. Université de Turku.

OCDE (2011) : Regards sur l'éducation. Les indicateurs de l'OCDE. Consultable en ligne sur : http://www.oecd-ilibrary.org/education/education-at-a-glance_19991487

PELTONEN T. (2002) : Pienten koulujen esiopetuksen kehittaminen - entisajan alakoulusta esikouluun. [L'amélioration de l'éducation préscolaire dans les petites écoles. Passé et présent]. Thèse de doctorat E60. Université d'Oulu.

PEPA (2011) : Aluehallintovirastojen keskeiset arviot peruspalvelujen tilasta 2010. PohjoisSuomen aluehallintoviraston julkaisuja 2011. [Rapport national d'évaluation sur l'état des services publics de base 2011]. Office régional de l'administration du Nord de la Finlande. Disponible en ligne sur: http://www.avi.fi/fi/virastot/pohjoissuomenavi/ Ajankohtaista/julkaisut/Documents/2011/AVI\%20peruspalvelut\%202010LR.pdf

PSAVI (2011): Pohjois-Suomen aluehallintoviraston alueen perusopetuksen ja lukiokoulutuksen tunnuslukutiedot vuodelta 2010. Pohjois-Suomen aluehallintoviraston julkaisuja 2011. [Statistiques annuelles de l'enseignement obligatoire et de l'enseignement secondaire du Nord de la Finlande 2010]. Office régional de l'administration du Nord de la Finlande. Disponible en ligne sur: http://www.laaninhallitus.fi/lh/ oulu/siv/home.nsf/pages/6865CBB82FE6B81BC225792600327FD4?opendocument

Statistics Finland (2011) : National statistics centre database. Consultable en ligne sur : http://www.tilastokeskus.fi/index.html 
